Madame Maria-José Aguilera Arilla

Madame María-Pilar Gonzalez

Yanci

Monsieur Vicente Rodriguez

Rodriguez

\title{
La nouvelle immigration polonaise en Espagne
}

In: Revue européenne de migrations internationales. Vol. 12 N¹. Espagne, Portugal, Grèce, pays d'immrigration. pp. 91-107.

Citer ce document / Cite this document :

Aguilera Arilla Maria-José, Gonzalez Yanci María-Pilar, Rodriguez Rodriguez Vicente. La nouvelle immigration polonaise en Espagne. In: Revue européenne de migrations internationales. Vol. 12 №1. Espagne, Portugal, Grèce, pays d'immrigration. pp. 91-107.

doi : 10.3406/remi.1996.1499

http://www.persee.fr/web/revues/home/prescript/article/remi_0765-0752_1996_num_12_1_1499 


\section{Résumé}

La nouvelle immigration polonaise en Espagne

María-José AGUILERA ARILLA, María-Pilar GONZALEZ YANCI, Vicente RODRIGUEZ RODRIGUEZ

Les anciens pays socialistes européens sont devenus, au cours de ces dernières années, des foyers d'émigration, avec des flux de départ significatifs, même si les niveaux prévus il y quelques années $\mathrm{n}$ 'ont pas été atteints. D'autre part, les pays méditerranéens, anciens foyers d'émigration, se sont transformés, depuis peu, en aires réceptrices. En Espagne, la moitié des immigrants en provenance des pays de l'Est sont des Polonais.

La population polonaise constitue en Espagne un groupe réduit, mais en cours d'accroissement. Les immigrants viennent tant du milieu rural que des principales villes de Pologne. Ils résident à Madrid et dans ses environs, et aussi dans les provinces côtières du Nord et de l'Est, les plus développées. C'est actuellement une immigration de caractère économique. Mais le groupe est encore marqué par l'ancienne immigration politique, dont le niveau de culture et de qualification professionnelle était élevé, ce qui lui a donné une image très positive, qui favorise encore aujourd'hui le bon accueil du groupe par la population espagnole.

\section{Abstract}

Polish New immigration in Spain

María-José AGUILERA ARILLA, María-Pilar GONZALEZ YANCI, Vicente RODRIGUEZ RODRIGUEZ

On one hand, the former socialist countries of Europe became country of emigration during these last years with some significant emigration flow, even if these one not reached the estimation. On the other hand, mediterranean countries changed to country of emigration. In Spain, half of immigrants from East countries are Polish.

Polish immigrants in Spain constitute a small group which is on the increase and which comes from both the rural and the main urban areas in Poland. They usually reside not only in Madrid and the metropolitan surrounding, but also in some coastal provinces in the east and the North, all of which are economically strong areas. The reasons for this new flow are currently considered to be economic, although, several years ago, Poles entered Spain as refugees or asylum-seekers. Their high academic level and professional qualification contribute to their good image and wide acceptance among Spanish society.

\section{Resumen}

La nueva inmigración polaca en España

María-José AGUILERA ARILLA, María-Pilar GONZALEZ YANCI, Vicente RODRIGUEZ RODRIGUEZ

Los antiguos países socialistas europeos han sido, en los últimos arios, importantes focos de emigración, aunque menos que la esperada en un principio. Por otro lado, los países del ambito mediterraneo, otrora focos de emigración, se han convertido recientemente en areas receptoras. En España, más del 50 \% de los inmigrantes procedentes de los países del Este son polacos.

La población polaca inmigrante es un grupo pequeño, pero en aumento, que procede tanto del medio rural como de las principales ciudades de Polonia. Residen en Madrid y su entorno más próximo, así como en varias provincias costeras del Norte y Este, todas ellas de desarollo económico importante. Es actualmente una inmigración de carácter económico, aunque empezó con una motivación política, con rasgos propios, como un nivel cultural y calificación profesional considerables, que favorecen su buena imagen y acogida entre la población española.

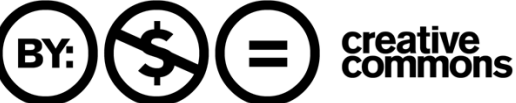


Revue Européenne des Migrations Internationales Volume $12-\mathrm{N}^{\circ} 1$ 1996

\title{
La nouvelle immigration polonaise en Espagne
}

\author{
María-José AGUILERA ARILLA \\ María-Pilar GONZALEZ YANCI \\ Vicente RODRIGUEZ RODRIGUEZ
}

Parmi les multiples changements qui se sont produits, tout au long de ce siècle dans les mouvements migratoires internationaux, se détachent ceux qui affectent le continent européen. Les aspects les plus significatifs constatés ces dernières années ont été la transformation des pays méditerranéens, qui de pays d'émigration sont devenus pays d'immigration, recevant principalement des Africains, et la reprise des flux migratoires de l'Est vers l'Ouest, interrompus durant la longue période des régimes communistes par ce que l'on a appelé le «rideau de fer».

La chute du mur de Berlin marque, de façon symbolique, le recommencement de ce mouvement, qui a de vieilles racines. On a d'abord beaucoup spéculé, en pronostiquant une possible avalanche d'émigrants, provenant des pays de l'Est vers l'Occident plus développé (STRAUBHAAR, 1992), et il y a même eu, de la part de certains gouvernements, un durcissement de la réglementation concernant le droit d'asile et le statut de réfugié. A la fin de 1995, on peut affirmer que ce mouvement migratoire de l'Est vers l'Ouest est réel, mais aussi qu'il reste dans des limites tout à fait normales et prévisibles après cette fermeture prolongée des frontières (SALT, 1992).

Il y a des raisons objectives variées qui impulsent ce mouvement. Comme le met en relief le rapport réalisé par la Commission des Communautés Européennes (COMISIÓN C.E. 1991), l'économie des pays de l'Est connaît de multiples problèmes, qui incitent la population à émigrer : haut pourcentage d'emploi industriel, mais dans un secteur obsolète et en crise, proportion excessive de personnes employées dans l'agriculture, avec de faibles perspectives d'avenir, mauvais état des infrastructures de transport et graves déséquilibres régionaux. Des facteurs psychologiques, une haute idée des conditions de vie occidentales, l'espoir de changements, 
etc... viennent renforcer ces facteurs économiques. Enfin la situation politique de certains des pays, qui connaissent de graves affrontements contribue à la reprise des flux. Le bon accueil qui, en général, est fait dans les pays occidentaux à cette population émigrante, culturellement proche et bien formée avant son départ, favorise le prolongement du processus, qui est entretenu par la difficulté à résoudre les problèmes ethniques et ceux des minorités dans les pays de l'Est, et par la longue durée probable de la période de transition politique et économique, qui à son tour, engendrera une augmentation du chômage (SALT, 1992).

C'est dans ce contexte que se place l'apparition en Espagne d'un courant d'immigrants en provenance des pays de l'Est, parmi lesquels les Polonais se détachent tout spécialement. L'objectif de ce travail est de faire une analyse des caractéristiques démographiques, socio-économiques et de la distribution spatiale de ce nouveau groupe de migrants.

\section{L'ESPAGNE, UN LIEU D'ACCUEIL DE L'ÉMIGRATION POLONAISE}

Le phénomène migratoire de la population polonaise est intimement lié à son destin historique. Quelques rapports (SOPEMI, 1982) mettent en évidence la complexité des mouvements migratoires, et surtout les difficultés pour les quantifier, liée essentiellement à la coexistence de flux légaux et illégaux. Le rapport du SOPEMI , se fondant sur les données du Bureau Central de Statistiques Polonais, évalue à 1093600 les émigrants en règle entre 1951 et 1990, parmi lesquels 271000 ont quitté le pays entre 1980 et 1990 . Cependant, d'autres estimations donnent, pour ces dix dernières années, un chiffre de 1100000 départs au total, en prenant en compte ceux qui sortent de façon illégale, ce qui aboutit à multiplier par quatre le chiffre précèdent. S'il en est ainsi, l'immigration illégale serait beaucoup plus importante que l'immigration légale.

Reprenant l'analyse de M. J. MARRON (1992), on peut distinguer trois étapes dans l'histoire de l'émigration polonaise au $\mathrm{XX}^{\mathrm{e}}$ siècle. La première, avant l'établissement du communisme, se caractérise par des flux de sortie permanents, mais de volume limité. La seconde, pendant la période communiste, est celle de l'interdiction de l'émigration en dehors des regroupements familiaux; le nombre des émigrants se réduit pour l'essentiel à ceux qui partent du pays de façon illégale. Pendant la troisième étape, depuis la chute du régime communiste en 1989, le volume de l'émigration augmente à nouveau, sans atteindre le niveau que certains avaient pronostiqué : MARRON parle de plus d'un million de sorties.

Dans le passé, la population polonaise émigrante, régulière ou irrégulière, s'était dirigée fondamentalement vers l'Allemagne de l'Ouest (58\% pour la première, $50 \%$ pour la seconde) et vers les Etats-Unis (10\% et $14 \%)$. L'Italie et l'Autriche, prises conjointement, seraient le lieu élu par 8 à $10 \%$ du total des émigrants légaux et illégaux. Ces pays et d'autres comme la France, le Royaume-Uni et la Belgique, comptent des communautés polonaises très nombreuses, dues à une émigration plus ancienne puisque l'on estime qu'entre 1860 et 1890 plus de 3,5 millions de Polonais abandonnèrent leur patrie. Les Etats-Unis à eux seuls ont reçu, entre 1860 et 1940 , presque deux millions d'émigrants polonais. 
Il n'est pas facile de préciser le nombre d'immigrants polonais établis en Espagne, parce qu'un pourcentage considérable d'entre eux n'y est pas comptabilisé du fait de l'illégalité de leur situation. Selon les estimations consulaires, ils pourraient être au total quelques 15000 .

A partir des sources officielles existantes on peut affirmer qu'il s'agit d'un groupe peu nombreux, mais en expansion, et occupant la première place dans l'immigration en provenance des pays de l'Est de l'Europe. Selon l'Institut National de la Statistique, il y avait en 1991 en Espagne plus de 3.000 résidents polonais, ce qui suppose plus de $50 \%$ du total du contingent des pays de l'Est, mais seulement $1 \%$ de celui des étrangers recensés cette année là (393 100). Il s'agit d'une population familiale bien établie. En 1992, ont été accordés 3276 permis de travail à des Polonais, chiffre à peu près semblable aux 3328 qui obtinrent la régularisation de leur situation la même année.

Ces sources montrent que cette population est en augmentation, et entre en Espagne pour des raisons essentiellement économiques, à l'opposé de celles des années antérieures, qui, du fait de la politique des pays de l'Est, tentait d'y accéder par le canal de la demande d'asile. Aujourd'hui l'Espagne tente de restreindre drastiquement ce type d'entrées, et l'on constate une diminution notable des demandes, comme des attributions du statut de réfugié. De 3279 Polonais qui en 1990 ont demandé l'asile politique, on est passé à 972 en 1991.

Les immigrants polonais établis en Espagne sont perçus avec un respect et une considération qui les différencie d'autres groupes étrangers immigrants. L'image que les Espagnols ont des Polonais est très positive. Plusieurs indices permettent de l'affirmer. Rarement ils apparaissent dans les rubriques de faits divers des journaux, et, à l'exception de quelques accusations de trafics illégaux (voitures notamment); ils ne sont pas accusés d'infractions, ni d'actions violentes, ou de participation à des affrontements avec d'autres groupes, qu'ils soient espagnols ou étrangers. Par contre, ils apparaissent dans d'autres rubriques, et l'on note que leurs voisins les apprécient, les considèrent comme sérieux et travailleurs, même s'ils reconnaissent avoir peu parlé avec eux parce qu'ils ne comprennent pas encore l'espagnol.

Si l'on cherche deux qualificatifs qui définissent avec clarté le caractère de l'immigrant polonais établi en Espagne, cela serait «intégrés et catholiques» (BERLIN, 1994), ou peut-être mieux encore catholiques et intégrés. En réalité, s'ils apparaissent comme intégrés dans la population espagnole, cela est dû, sans doute, à diverses raisons outre celle d'être catholiques et donc de participer à une culture religieuse semblable à celle du peuple qui les accueille. Ils sont blancs, évidemment, la couleur de leur peau n'est pas un élément de rejet pour la population autochtone. Ils ont un niveau culturel relativement élevé comme l'a mis en relief une enquête réalisée en 1993, qui a révélé que le quart d'entre eux avaient fréquenté une université, $55 \%$ étaient bacheliers et que $20 \%$ seulement n'avaient pas terminé leurs études élémentaires. Cinq années auparavant, cependant, la proportion de ceux qui avaient poursuivi des études supérieures était encore plus élevée, atteignant $42 \%$, ce qui a contribué à persuader les Espagnols que les Polonais étaient des gens cultivés, et a forger leur excellente image actuelle. 
Leur qualification professionnelle est aussi reconnue, il suffit d'observer que, dans les petites annonces destinées à demander un emploi, ils sont parmi les rares étrangers, qui en même temps que leur métier, indiquent leur nationalité, car ils savent que leur annonce va être ainsi valorisée. Les indications comme plombier polonais, maçon polonais, serrurier polonais sont fréquentes dans les annonces de la presse gratuite. Ils passent aussi pour entreprenants et surtout sérieux, tout au moins dans l'opinion de nombreuses personnes qui ont utilisé leurs services, et qui en attestent. De plus, et pour finir, ils sont solidaires (comme c'est le cas pour d'autres groupes d'émigrants, bien que pas pour tous), car ceux qui sont déjà établis en Espagne aident, dans les premiers temps, ceux qui arrivent, selon les déclarations des représentants consulaires.

\section{CARACTÉRISTIQUES GÉNÉRALES DES IMMIGRANTS POLONAIS}

\section{ORIGINE ET DESTINATION}

L'analyse des lieux d'origine des Polonais n'est pas une tâche simple. On peut tenter de l'aborder à travers le dépouillement du Registre Consulaire qui ne dispose d'informations que sur les 982 résidents polonais inscrits, effectif très inférieur à celui obtenu à partir des sources officielles espagnoles. Grâce aux fichiers consulaires nous connaissons pour chacune de ces personnes le lieu d'origine (la «voïvodie», unité administrative équivalente à la province espagnole ou au département français, la ville et l'adresse postale), ainsi que l'adresse en Espagne.

La carte (figure 1) montre que la majorité des immigrants viennent des zones rurales du sud du pays, loin du nord industriel et urbanisé. Près de $30 \%$ d'entre eux viennent des voïvodies situées au Sud-Est de la Pologne, et c'est là que se situe la voïvodie de Rzeszow, lieu d'origine à elle seule de $13,5 \%$ des migrants. La deuxième région de départ est le sud-ouest, avec $15 \%$ des départs. Les autres lieux d'origine jouant un certain rôle sont plus dispersés : au total $13 \%$ des immigrants en Espagne sont originaires des trois aires urbaines les plus importantes de la Pologne, Varsovie, Katowice et Lodz. Dans le reste de la Pologne seule la frange Nord, bordant la Baltique, compte un nombre significatif de départs enregistrés.

Le schéma de distribution dans l'espace espagnol de ces Polonais enregistrés au Consulat est très proche de celui que l'on peut observer, pour un plus grand nombre d'immigrants, donc avec une meilleure fiabilité, à travers les sources statistiques espagnoles. Deux cartes ont été élaborées, l'une à partir des adresses données lors du processus de régularisation de 1991, l'autre avec celles fournies lors de l'établissement des permis de travail en 1992 (figure 2). Ces cartes indiquent que presque toutes les provinces espagnoles possèdent dans leur population, des immigrés polonais. On y lit aussi la très forte concentration d'immigrants à Madrid : cette province regroupe à elle seule plus de la moitié des Polonais pour chacune des deux sources utilisées. L'autre moitié se distribue de façon dispersée entre presque toutes les provinces espagnoles, même si l'on observe des groupes plus nombreux dans certaines zones, surtout au centre et sur le littoral oriental. 


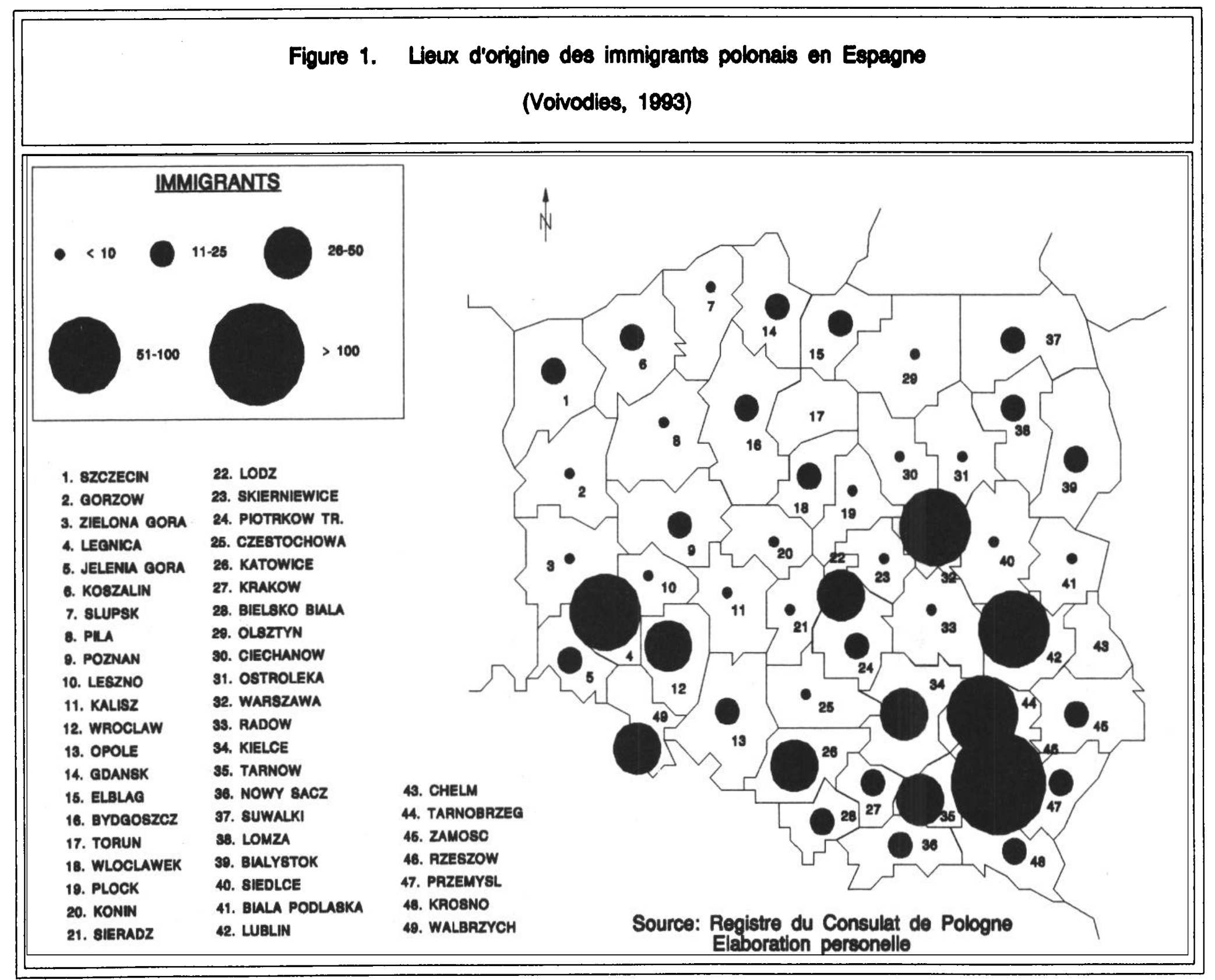


Figure 2. Les immigrants polonais en Espagne

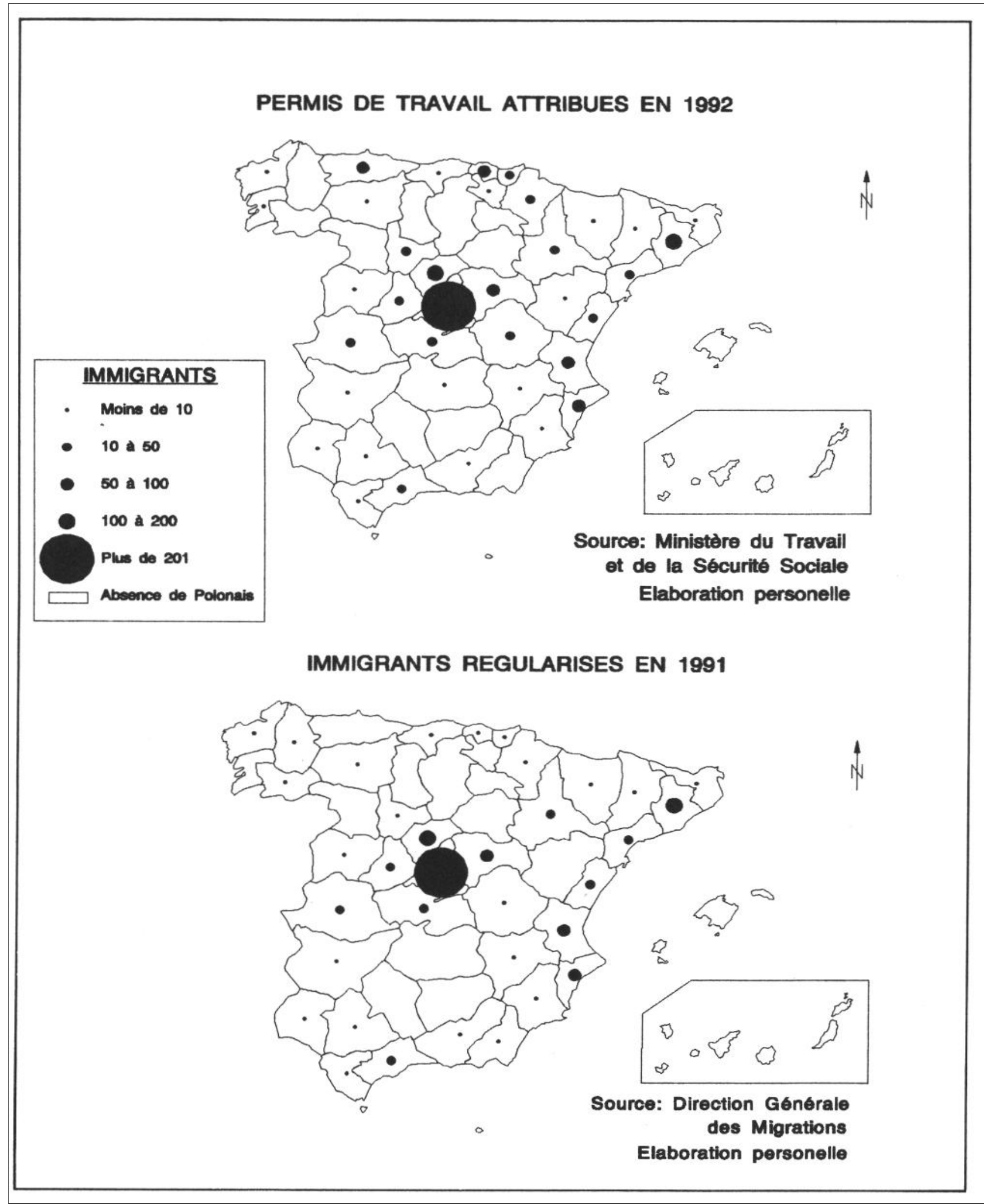


Au delà de ces similitudes, des différences importantes existent entre les deux sources. Elles sont liées en particulier au fait que, lors de la régularisation, près du quart des Polonais n'ai pas précisé dans quelle province espagnole il vivait. Cette non-déclaration s'explique peut-être par la situation encore peu stabilisée de beaucoup de migrants, qui peuvent ainsi s'être rapprochés de Madrid et de la Direction Générale des Migrations pour déposer leur dossier de régularisation. Par contre, au moment où ils demandent leur permis de travail ils ont une adresse dans la province dans laquelle ils ont l'intention d'exercer l'emploi pour lequel ils ont demandé ce permis.

Cette forte mobilité explique les écarts entre les deux cartes. Selon la source, les chiffres par province peuvent varier beaucoup. Dans sept des provinces aucun Polonais n'a été régularisé. Pourtant, dans quatre d'entre elles, des Polonais ont déposé des demandes de permis de travail quelques mois après la régularisation. A l'inverse, dans quatre autres provinces, on observe des régularisations, mais aucune demande de permis de travail.

Au-delà de ces écarts qui témoignent de la faible fiabilité des sources statistiques disponibles, la distribution spatiale des immigrants polonais en Espagne répond à un schéma centre-littoral. Le volume de migrants le plus important se situe autour de Madrid et apparaît très influencé par la capitale. D' autre part, sur le littoral, leur localisation correspond à la distribution des aires les plus peuplées, celles qui ont le meilleur niveau économique et sont le mieux desservies. Ce sont aussi les provinces qui proposent l'offre de travail la plus large.

Près des trois quarts des immigrants polonais recensés en 1991 (Recensement de la Population) vivent dans la province de Madrid. Ici, l'exploitation particulière menée par le Département d'Economie de la Communauté Urbaine (voir note 1) permet d'observer leur distribution à une échelle plus fine, intra-provinciale et municipale. La carte (figure 3) permet de distinguer les principales zones de concentration, l'axe de la route V, Madrid-Estramadoure, au sud-est de la métropole, qui regroupe $54 \%$ des Polonais, et l'axe de la route nationale II, Madrid-Barcelone, au nord-est, qui en réunit $21 \%$. Dans les deux cas il s'agit de zones de résidence d'une population au niveau économique bas. Par ailleurs, $20 \%$ des Polonais habitent la capitale elle-même tandis que les $5 \%$ restants habitent d'autres communes de la province.

En dehors de ces espaces de concentration, 46 communes, (une commune de la province sur quatre) comptent quelques immigrants polonais parmi leurs habitants. Ces communes sont celles où commence à se faire sentir l'influence métropolitaine.

La distribution par districts des immigrants polonais qui résident à Madrid même (carton de la figure 3), est peu significative étant donnée la faiblesse des effectifs. Seuls les districts de Centro et Latina, situés dans le centre urbain et très dégradés, se détachent par leur relative importance (autour de 30 Polonais).

Cette distribution spatiale peut s'expliquer par la coïncidence de l'arrivée de ces immigrants, et de la croissance de ces communes de l'aire métropolitaine de Madrid, avec une offre de logements moins coûteux que ceux de la capitale. 


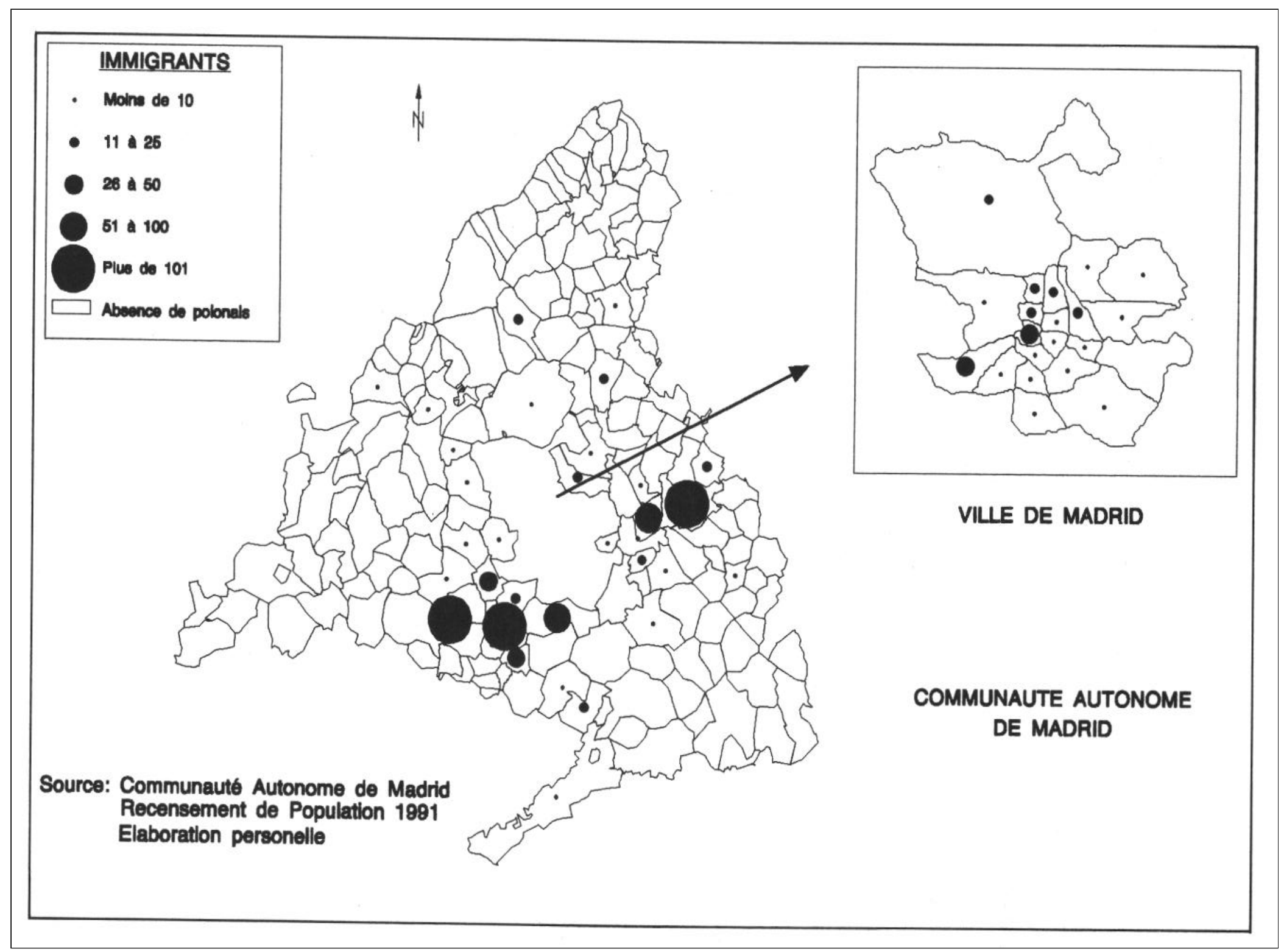

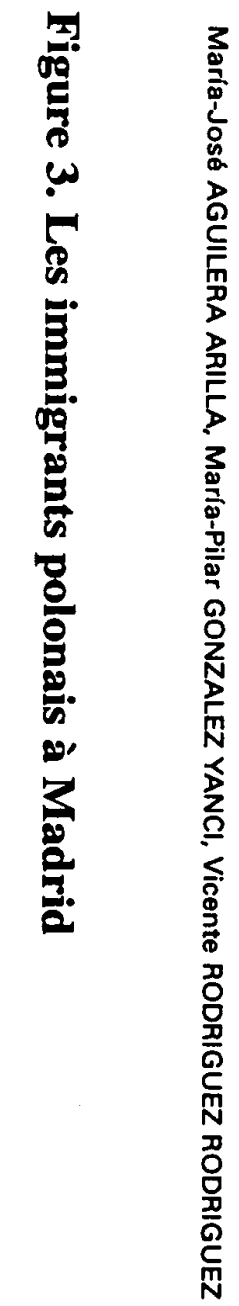




\section{LES CARACTÈRES DÉMOGRAPHIQUES}

Etant donné la proportion importante des immigrants polonais vivant à Madrid, l'analyse démographique de ses résidents donne une idée de cette population que l'on doit pouvoir généraliser à toute l'Espagne. Elle présente tous les caractères que l'on retrouve dans les migrations de type économique : population d'âge actif, fortement sur-masculine.

Une première pyramide des âges a été construite grâce à l'exploitation particulière du recensement, qui permet de prendre en compte l'ensemble du groupe familial (figure 4). Elle permet de mettre en évidence les traits suivants :

- une claire prédominance des adultes dont les deux tiers ont entre 20 et 40 ans, avec un coefficient de masculinité de 138 hommes pour 100 femmes.

- une présence significative de jeunes de moins de 20 ans (20\% de l'ensemble), ce qui démontre la présence de familles, le plus souvent des couples avec un ou deux enfants, pas très différentes de la famille madrilène type.

- un faible pourcentage de personnes âgées (à peine 1,5\%), plus faible que celui que l'on observe dans les autres populations venues des pays de l'Est, et qui contraste notablement avec d'autres groupes d'Européens résidant à Madrid et en Espagne ${ }^{2}$, et démontre encore une fois le caractère économique de l'immigration polonaise.

Avec ces caractéristiques, l'indice de dépendance est très faible, moins de 25 «dépendants» (enfants et personnes âgées) pour 100 adultes, ce qui facilite à la fois une forte participation à l'activité et une grande mobilité géographique.

Dans cette population bien implantée, les célibataires sont à peine plus nombreux que les individus mariés, avec une certaine différence selon le sexe. Les femmes mariées sont plus nombreuses, mais c'est l'inverse pour les hommes. C'est à nouveau un phénomène en relation avec le caractère économique de la migration, la place importante des hommes jeunes et célibataires, groupe le plus dynamique et ayant les plus grandes possibilités d'adaptation au marché de l'emploi.

L'analyse d'autres sources donne un nouvel éclairage sur l'immigration polonaise. Le Recensement de la Population de 1991 ne nous renseigne que sur une partie du groupe, précisément celle qui est le mieux établie. En revanche, les dossiers de la régularisation nous informent sur les caractéristiques des derniers arrivés et, pour les actifs, sur leur type de permis de travail.

Ces données, contrairement aux précédentes, englobent tout le territoire national, et montrent que l'aspect le plus marquant est l'absolue prédominance d'hommes plutôt jeunes.

La pyramide de la population des régularisés (figure 4) est fortement déséquilibrée en faveur des hommes, ceux de 20-29 ans en particulier. De plus, la majorité de la population ( $54 \%$ ) est constituée de célibataires. Ces caractéristiques se retrouvent dans les données sur les permis de travail. Ils sont obtenus par des hommes dans $78 \%$ des cas, jeunes pour la plupart, puisque seulement $1 \%$ d'entre eux a plus de 55 ans, tandis que la tranche d'âges des $20-24$ ans en regroupe $24 \%$. 
Figure 4. Les Polonais de Madrid : structure par sexe et par âge

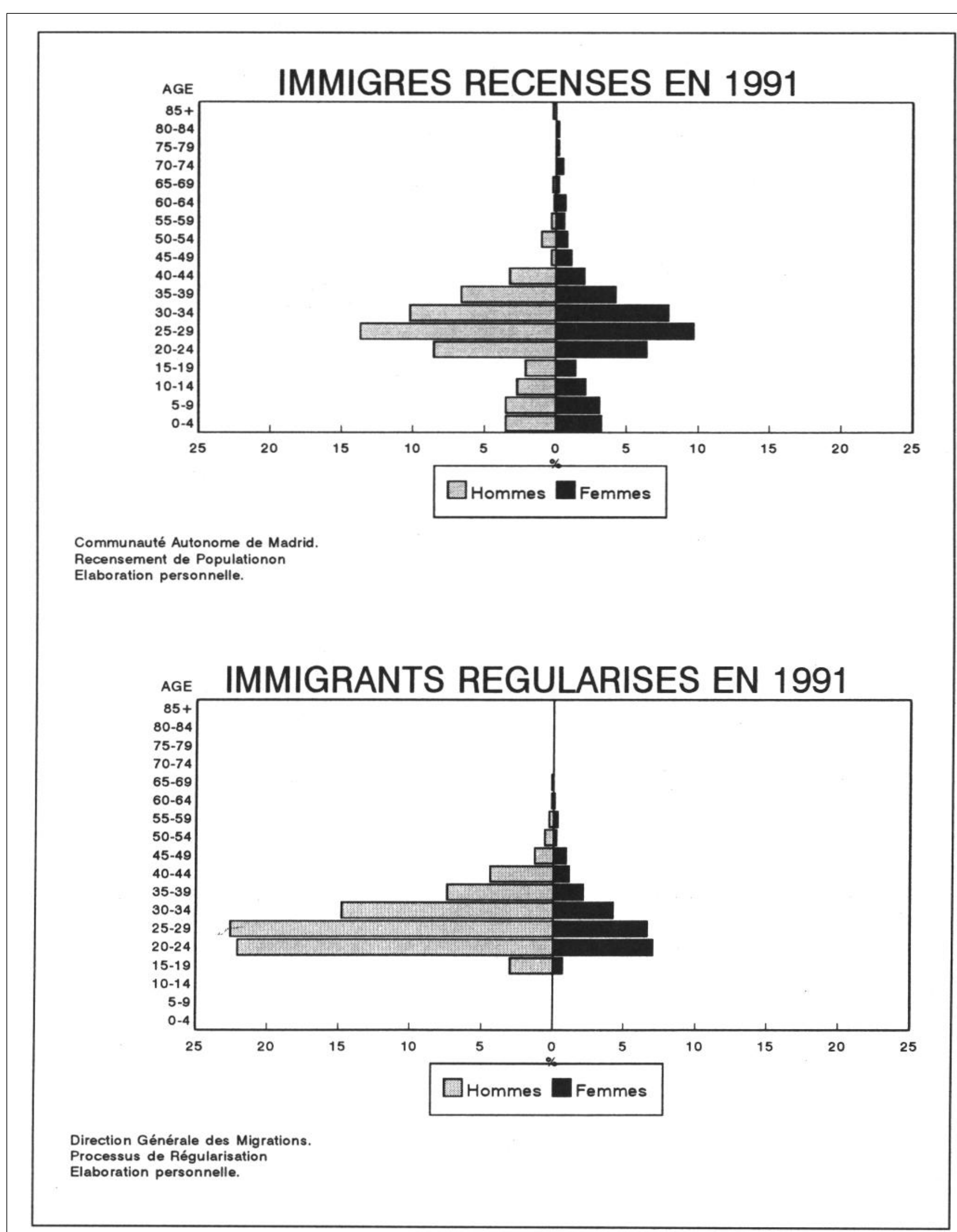


En conclusion, les Polonais forment bien en Espagne une population encore majoritairement masculine, jeune et célibataire, mais parmi les plus anciennement implantés, des familles se sont constituées, et leur nombre tend à s'accroître.

\section{LE MONDE DU TRAVAIL}

Comme dans toutes les populations migrantes, on note chez les Polonais de fortes différences entre les hommes et les femmes dans le domaine de l'emploi.

Si l'on se réfère au recensement de 1991, unique source nous informant sur le taux d'activité, on constate que celui-ci est élevé : $49 \%$, niveau proche de la moyenne espagnole, mais avec des différences appréciables selon les sexes. Pour les hommes le taux est de $60 \%$, pour les femmes il atteint $40 \%$ ce qui confirme la place acquise par la travailleuse polonaise immigrante en tant qu'élément fondamental de l'économie familiale.

Tableau : Les immigrants polonais et l'activité Communauté Autonome de Madrid

\begin{tabular}{|l|r|r|r|r|}
\hline \multirow{2}{*}{} & \multicolumn{2}{|c|}{ Hommes } & \multicolumn{2}{c|}{ Femmes } \\
\cline { 2 - 5 } & effectifs & \multicolumn{1}{|c|}{$\%$} & effectifs & \multicolumn{1}{c|}{$\%$} \\
\hline ENSEMBLE & 563 & 56,0 & $\mathbf{4 4 3}$ & $\mathbf{4 4 , 0}$ \\
\hline ACTIFS & 326 & 58,1 & 167 & 37,7 \\
dont & & & & \\
actifs ayant un emploi & 238 & 66,1 & 122 & 33,9 \\
Chômeurs n'ayant encore jamais travaill & 65 & 69,9 & 28 & 30,1 \\
Autres chômeurs & 23 & 57,5 & 17 & 42,5 \\
\hline INACTIFS & 237 & 46,2 & 276 & 53,8 \\
dont & & & & \\
Enfants & 107 & 64,5 & 59 & 35,5 \\
Etudiants & 114 & 53,0 & 101 & 47,0 \\
Personnes au foyer & 7 & 6,1 & 108 & 93,9 \\
Retraités et pensionnés & 9 & 52,9 & 8 & 47,1 \\
\hline
\end{tabular}

Source : CAM, Recensement de Population, 1991, élaboration personnelle

Cependant, ni le type d'activité ni les périodes de travail ne sont stables, dans le contexte de l'économie espagnole, qui depuis 1992 traverse une crise importante. La preuve en est donnée par le taux élevé du chômage chez les immigrants polonais (27\%) au recensement en mars 1991. Nous ne pouvons pas mesurer son évolution depuis cette époque, ni l'ampleur de l'emploi non déclaré, au noir, dont on sait qu'elle est l'importance chez les immigrants.

L'examen des professions déclarées par les immigrants polonais au moment de la demande de permis de travail ou de la régularisation, et celui de la branche d'activité à laquelle se rattache l'entreprise qui les emploie, permet d'établir la relation 
entre leur qualification personnelle et l'activité économique à laquelle ils participent en Espagne.

La distribution des immigrés polonais par branche d'activité nous est connue. Les différentes sources donnent en effet des résultats semblables : plus de la moitié d'entre eux travaillent dans des entreprises de services, un tiers dans le bâtiment, le reste dans des entreprises industrielles (un peu plus de $12 \%$ ) et de l'agriculture (autour de $3 \%$ ).

Une analyse plus détaillée des professions des actifs employés dans le bâtiment indique que parmi ces hommes on rencontre des maçons et des manoeuvres du bâtiment, à faible qualification, et des électriciens, des monteurs, des chauffeurs et des conducteurs d'engins, ayant une qualification plus élevée. Les qualifiés occupent presque toujours des emplois en relation avec leur qualification (industrie, construction ou transports). On les trouve surtout dans les régions urbaines de Madrid, Barcelone, Valence et les Asturies, là où l'offre de travail est suffisante pour leur permettre de mettre en valeur leur qualification, de se voir reconnaître comme de bons professionnels par la population autochtone. Les non-qualifiés, que l'on rencontre dans les mêmes espaces, ont aussi souvent déclaré une profession en relation avec le travail qu'ils réalisent. Cette activité est un peu mieux représentée chez ceux qui ont obtenu leur permis de travail par la procédure normale que chez les régularisés.

Dans l'emploi relevant du secteur tertiaire la situation est plus complexe. Les services domestiques emploient $18 \%$ des régularisés et $30 \%$ de ceux qui ont sollicité un permis de travail, et la proportion est plus élevée à Madrid. C'est une activité exercée quatre fois sur cinq par les femmes. Bien que la qualification ne soit pas nécessaire dans les activités du service domestique, les femmes polonaises ont une réputation de sérieux et de qualité du travail, malgré des difficultés linguistiques qui n'apparaissent pas comme un obstacle insurmontable.

Dans l'hôtellerie et la restauration, sous-secteur qui emploie $10 \%$ des immigrés polonais, le niveau de qualification est plus élevé. Une grande partie des employés ont reçu une formation à ces métiers, qui correspondent à la profession qu'ils ont déclarée. En plus des zones urbaines, on les rencontre aussi dans quelques provinces méditerranéennes (Baléares, Valence) où il y a une demande dans ce domaine, liée à l'importance des activités touristiques.

Les rares immigrants polonais qui travaillent dans l'agriculture sont en grande majorité des hommes qui avaient une qualification préalable dans ce domaine. On les rencontre dans les provinces agricoles du Levante (Valencia, Castellón) et du centre de l'Espagne (Cáceres, Segovia). Ils ne représentent un pourcentage significatif (4\%) que parmi les immigrants qui ont bénéficié du processus de régularisation. L'offre d'emploi dans ce secteur en Espagne, spécialement pour le travail temporaire, explique probablement cette situation.

Par contre un des traits majeurs qui qualifie l'immigration polonaise plus anciennement établie en Espagne, c'est le pourcentage notable de membres des professions libérales, spécialement au Pays Basque, en Navarre et à Madrid. L'on ne note pas de spécialisations professionnelles particulières, et l'on peut noter un équilibre entre les deux sexes. Les classes moyennes sont, elles, peu représentées, soit que les Polonais 
se heurtent aux obstacles de la langue et de la méconnaissance du système administratif (employés de bureau, cadres administratifs), soit qu'ils entrent en concurrence avec d'autres groupes d'immigrants (commerçants).

Apparaît ainsi une dichotomie entre les travailleurs de basse et haute qualification, les premiers sont très nombreux, les seconds sont rares, mais atteignent des proportions qualitativement significatives si on compare les Polonais avec d'autres immigrants, notamment ceux qui viennent des pays en voie de développement, ce qui joue sans doute sur la bonne image sociale du groupe national.

C'est probablement leur niveau de scolarisation qui explique les différences entre les caractéristiques professionnelles des Polonais et celles des autres immigrants, en particulier de ceux qui viennent du Tiers Monde. C'est ainsi que parmi les immigrants polonais de Madrid, presque $60 \%$ ont suivi un enseignement secondaire, avec une proportion plus élevée pour les hommes que pour femmes. Il y a très peu d'analphabètes, en revanche près du tiers d'entre eux a poursuivi des études supérieures, chez les hommes comme chez les femmes en raison de la généralisation en Pologne de l'enseignement supérieur. Cette formation de niveau secondaire ou universitaire conditionne la profession des immigrants et les oriente vers des branches différentes. C'est ainsi que, ceux qui ont le niveau du baccalauréat cherchent un emploi et déclarent une profession demandant peu de qualification, ouvriers du bâtiment ou de l'industrie, services domestiques. Par contre, les immigrants ayant suivi des études universitaires tendent à se déclarer comme cadres supérieurs (un tiers du total), mais on les rencontre aussi exerçant des professions moins qualifiées (industrie, services domestiques), et dans les activités commerciales. Ceci démontre que même les immigrants les plus qualifiés ne trouvent pas toujours à Madrid des situations correspondant à leur niveau de formation, qu'il y a un processus de sous-utilisation de leur capacité professionnelle qui a été bien analysé dans d'autres pays récepteurs de l'immigration polonaise, comme par exemple l'Autriche (FASSMAN, KOHLBACHER et REEGER, 1993). Mais il est difficile d'affirmer que cette description soit aujourd'hui applicable à toute l'Espagne.

La majorité des Polonais travaillant en Espagne (94\%) sont salariés. Dans le groupe réduit des patrons, de ceux qui se sont installés à leur compte, on retrouve les mêmes branches d'activité que chez les salariés : $72 \%$ des entreprises relèvent du bâtiment, des services aux entreprises et des services domestiques. Elles sont implantées dans les aires urbaines, où pourtant la main d'oeuvre autochtone ne manque pas, mais les petits entrepreneurs polonais, bien formés, ont su prendre une certaine part du marché. Ils sont appréciés à cause de leur habilité et leur qualification, mais aussi parce qu'ils développent des formes de travail nouvelles comme le travail à l'heure, ou l'organisation d'équipes qui réalisent des tâches complémentaires en maçonnerie, plomberie, etc.

Les salariés aspirent à entrer dans les entreprises industrielles, de services ou de distribution, pour bénéficier de contrats de longue durée. Par contre, dans le bâtiment la précarité est plus fréquente, à cause du caractère temporaire de l'activité, mais aussi du caractère instable des petites entreprises qui emploient les immigrants. 
Les permis de travail des immigrants polonais sont presque toujours des permis d'un an (plus de $95 \%$ ), avec un taux élevé de renouvellement (plus de $51 \%$ ). Les permis de court séjour sont exceptionnels. Ceux de longue durée, très rares aussi, ont été attribués à Madrid, au Pays Basque et aux Asturies; sans doute à des cadres supérieurs et des techniciens très qualifiés de l'industrie et des services aux entreprises de ces régions.

L'émigration polonaise vers l'Espagne a donc évolué; clairement politique à l'origine, elle offre aujourd'hui des caractéristiques économiques semblables à celles de la plupart des groupes nationaux que l'Espagne accueille. Mais son passé explique des particularités qui font la singularité de ce groupe immigré.

Traduit de l'espagnol par Jean-Claude SOUCHAUD, Doctorant en Géographie, MIGRINTER, Université de Poitiers 


\section{Notes et références bibliographiques}

(1). Les sources utilisées pour la réalisation de cette recherche sont :

- Le recensement (Censo de Población y Viviendas) de 1991, réalisé par l'Institut National de la Statistique et exploité par le Département d'Economie de la Communauté de Madrid. Grâce à cette exploitation on a disposé de renseignements sur les personnes de nationalité polonaise : lieu de résidence, âge, sexe, état civil, niveau d'études, activité, profession).

- Les statistiques sur les permis de travail attribués aux étrangers par le Ministère du Travail (1992). Cette source nous informe pour chaque actif sur son sexe, son âge, la province dans laquelle il vit, le type de permis dont il dispose, s'il occupe un emploi, le statut salarié ou non de cet emploi, son secteur et sa branche d'activité.

- Les statistiques issues du Processus de Regularisation, réalisé par la Direction Générale de Migrations del Ministère du Travail (1991). On a collecté alors des informations sur les étrangers qui ont sollicité la régularisation de leur situation (sexe, l'état-civil, distribution par provinces, profession, activité de l'entreprise leur ayant signé un contrat de travail, type de permis de travail qui leur a été délivré.

- Les statistiques sur l'asile et le refuge, qui fournissent très peu d'autres renseignements que la situation dans ce domaine.

- Le Registre Consulaire de Pologne n'a pas d'objectif statistique. Comme l'inscription n'est pas obligatoire elle ne prend pas en compte l'ensemble du groupe et son utilité est surtout de fournir un échantillon, sa dimension lui conférant une grande fiabilité. Son principal intérêt est d'apporter une information sur le lieu de provenance et de destination des immigrants. De plus, le Consulat est une source importante d'informations orales.

(2). On observe en Espagne une immigration de personnes âgées, non actives, qui viennent de pays riches de l'Europe, attirées par les conditions climatiques, et qui viennent passer leurs dernières années surtout dans les régions du littoral.

AGUILERA (M. J.), GONZALEZ YANCI (M. P.), RODRIGUEZ (V.), «Foreign immigrants in Madrid and the Metropolitan area : some general features», 27th International Geographical Congress, Washington, 1992.

BERLIN (B.), «Polacos : integrados y católicos», Rev. Carta de España n 478, febrero 1994.

COMISION C. E., «Aspectos socioeconomicos de los Estados y regiones de la Europa Central y del Este», Las regiones en la decada de los noventa. Cuarto informe periodico sobre la situación y la evolución socioeconomica de las regiones de la Comunidad, Bruxelles, 1991.

CHORAZYKIEWICZ (G.), MAREK (E.) «Poland and current international migration», Mass Migration in Europe. Implication in East and West, Vienna, 1992.

EUROSTAT, Pologne 1992, Monographie pays, Statistiches Bundesamt, 1992, 42 p.

FASSMANN (H.), KOHLBACHER (J.), REEGER (U.), Brain drain and brain waste. Skilled migration in Vienna, International Seminaire on Skilled and Highly Skilled Migration, Latina, 1993, 9 p.

GONZALEZ YANCI (M. P.), AGUILERA (M. J.), RODRIGUEZ (V.), «La inmigración extranjera en el espacio metropolitano de Madrid, Analisis de detalle de sus implicaciones», IV Jornadas de la Población Española, Inmigración extranjera y planificación demográfica en España, La Laguna, 1993, pp. 475-483.

HONEKOPP (E.), «Migratory mouvments from countries of Central and Eastern Europe», Council of Mouvments Europe Conference, Vienna, 1991.

KORCELLI (P.), «European migration : the polish example», Mass Migration in Europe. Implication in East and West, Vienna, 1992.

MARRON GAITE (M. J.), «Inserción laboral de los inmigrantes polacos en España durante el quinquenio 1987-1991», IVas Jornadas de la Población Española, Inmigración extranjera y planificación demográfica en España, La Laguna, 1993. 
RODRIGUEZ (V.), AGUILERA (M. J.), GONZALEZ YANCI (M. P.), «Foreign Minorities from Developping Countries in Madrid», GeoJournal, vol. 30, n³, 1993, pp. 293-300.

SALT (J.), «The future of international labor migration», International Migration Review, XXVI.4, pp. 1077-1111, 1992.

SOPEMI, «Mouvements migratoires en Europe Centrale et Orientale, le cas de la Hongrie, de la Pologne et de la République Fédérative Tchèque et Slovaque», Tendances des migrations internationales, Partie III, pp. 97-127, Paris, OCDE, 1992.

SOPEMI, Poland 1990, Paris, OCDE, 1992.

STRAUBHAAR (T.), «Allocational and distributional aspects of future immigration to Western Europe», International Migration Review, XXVI.2, 1992, pp. 462-483.

WORACH-KARDAS (H.), «Phases of life and migration in Poland», Mass Migration in Europe. Implication in East and West, Vienna, 1992. 
La nouvelle immigration polonaise en Espagne

María-José AGUILERA ARILLA, María-Pilar GONZALEZ YANCI, Vicente RODRIGUEZ RODRIGUEZ

Les anciens pays socialistes européens sont devenus, au cours de ces dernières années, des foyers d'émigration, avec des flux de départ significatifs, même si les niveaux prévus il y quelques années n'ont pas été atteints. D'autre part, les pays méditerranéens, anciens foyers d'émigration, se sont transformés, depuis peu, en aires réceptrices. En Espagne, la moitié des immigrants en provenance des pays de l'Est sont des Polonais.

La population polonaise constitue en Espagne un groupe réduit, mais en cours d'accroissement. Les immigrants viennent tant du milieu rural que des principales villes de Pologne. Ils résident à Madrid et dans ses environs, et aussi dans les provinces côtières du Nord et de l'Est, les plus développées. C'est actuellement une immigration de caractère économique. Mais le groupe est encore marqué par l'ancienne immigration politique, dont le niveau de culture et de qualification professionnelle était élevé, ce qui lui a donné une image très positive, qui favorise encore aujourd'hui le bon accueil du groupe par la population espagnole.

\section{Polish New immigration in Spain}

\section{María-José AGUILERA ARILLA, María-Pilar GONZALEZ YANCI, Vicente RODRIGUEZ RODRIGUEZ}

On one hand, the former socialist countries of Europe became country of emigration during these last years with some significant emigration flow, even if these one not reached the estimation. On the other hand, mediterranean countries changed to country of emigration. In Spain, half of immigrants from East countries are Polish.

Polish immigrants in Spain constitute a small group which is on the increase and which comes from both the rural and the main urban areas in Poland. They usually reside not only in Madrid and the metropolitan surrounding, but also in some coastal provinces in the east and the North, all of which are economically strong areas. The reasons for this new flow are currently considered to be economic, although, several years ago, Poles entered Spain as refugees or asylum-seekers. Their high academic level and professional qualification contribute to their good image and wide acceptance among Spanish society.

\section{La nueva inmigración polaca en España}

María-José AGUILERA ARILLA, María-Pilar GONZALEZ YANCI, Vicente RODRIGUEZ RODRIGUEZ

Los antiguos países socialistas europeos han sido, en los últimos años, importantes focos de emigración, aunque menos que la esperada en un principio. Por otro lado, los países del ambito mediterraneo, otrora focos de emigración, se han convertido recientemente en areas receptoras. En España, más del $50 \%$ de los inmigrantes procedentes de los países del Este son polacos.

La población polaca inmigrante es un grupo pequeño, pero en aumento, que procede tanto del medio rural como de las principales ciudades de Polonia. Residen en Madrid y su entorno más próximo, así como en varias provincias costeras del Norte y Este, todas ellas de desarollo económico importante. Es actualmente una inmigración de carácter económico, aunque empezó con una motivación política, con rasgos propios, como un nivel cultural y cualificación profesional considerables, que favorecen su buena imagen y acogida entre la población española. 